\title{
Abdominal Wall Blocks in Abdominal Surgery: An Update
}

\author{
TVS Gopal ${ }^{1}$, Amjad Maniar ${ }^{2}$, Arunangshu Chakraborty', Rammurthy Kulkarni ${ }^{2}$ \\ ${ }^{1}$ Department of Anaesthesia, Care Hospitals, Banjara Hills, Hyderabad, Andhra Pradesh, India. \\ ${ }^{2}$ Department of Anaesthesia, Axon Anaesthesia Associates, Bengaluru, India. \\ ${ }^{3}$ Department of Anaesthesia, Tata Medical Center, Kolkata, India.
}

\begin{abstract}
Abdominal wall blocks provide an alternative to the gold standard, epidural analgesia, as a part of multimodal analgesic regime for pain relief following a variety of abdominal surgeries. The ubiquitous availability of ultrasound and the paradigm shift to deposition of local anaesthetic into interfascial planes popularized abdominal wall blocks. Over the years, a better understanding of cadaveric, clinical sonoanatomy, and techniques have emerged. However, with certain abdominal wall blocks, spread of local anaesthetic is neither consistent nor predictable. This concise update presents details pertaining to ultrasound guided abdominal wall blocks.
\end{abstract}

\section{Introduction}

Pathology affecting vital organ systems in the abdomen often requires surgical correction. Pain after abdominal surgery, more so with laparotomy, is intense and is a combination of somatic and visceral components. Poor analgesia in the postoperative period affects respiration, triggers a sympathetic response, delays gut motility and mobilization [1]. The accepted gold standard for pain relief is continuous mid-to-low thoracic epidural analgesia, based on the extent of incision. Systemic opioids form the mainstay when an epidural is contraindicated, technically difficult to perform, or ineffective.

The utility of abdominal wall blocks dates back to 1899 when Schleich [2] first described rectus sheath block for relaxation during abdominal surgery. Thomas Seldon [3], in 1941, elucidated the nerve supply of the abdominal wall, local infiltration, and field block techniques for procedures of the abdomen. In 2001, Rafi [4], described his experience with an abdominal wall block via the inferior lumbar or Petit's triangle (Fig. 1). Ultrasound guided transversus abdominis plane block was first introduced into practice by McDonnell et al and Hebbard et al, both in 2007 [5, 6]. Thereafter, the widespread use of ultrasound guidance by regional anaesthesiologists led to modifications of the TAP block, precision with blocks such as ilioinguinal-iliohypogastric nerves and rectus sheath blocks, and introduction of newer blocks, viz. transversalis fascia plane block and quadratus lumborum block. These ultrasound guided blocks mostly provide somatic analgesia, with the exception of quadratus lumborum block that provides visceral analgesia also and are a component of the multimodal analgesia regime for pain relief after abdominal surgery.

\section{Relevant Anatomy}

For the practice of ultrasound guided regional anaesthesia (USGRA) one must be conversant with important structures and landmarks, all the while remembering that there may be anatomical variations often encountered with USGRA. The anterior abdominal wall, on either side of the midline, is covered by the rectus abdominis muscle (RAM), which is a vertically running five pairs of muscle on both sides of the midline, separated by a tendinous bridge known as the linea alba. The muscle is contained in the rectus sheath, which consists of the aponeuroses of the lateral abdominal muscles. Another tendinous line on the lateral border of RAM, linea semilunaris, separates RAM from the lateral abdominal wall muscles namely the external oblique (EOM), the internal oblique (IOM), and the transversus abdominis (TAM). Both the anterior and posterior rectus sheaths are distinct entities that are easily visualized on sonography. In the upper three quarters, the anterior rectus sheath is formed by the aponeurotic sheaths of the EOM and IOM. The posterior sheath is formed by the internal and transversus abdominis muscle. In

Address of Correspondence: Dr. TVS Gopal, Clinical Director, Care Hospitals, Banjara Hills, Hyderabad, Andhra Pradesh, India.

E-mail: tvsgopal@gmail.com

DOI: 10.13107/ijra.2021.v02i01.026 | C 2021 International Journal of Regional Anaesthesia | Available on www.ijrajournal.com | This is an Open Access article distributed under the terms of the Creative Commons Attribution Non-Commercial License (http://creativecommons.org/licenses/by-nc/3.0) which permits unrestricted non-commercial use, distribution, and reproduction in any medium, provided the original work is properly cited.

How to cite this article: Gopal TVS, Maniar A, Chakraborty A, Kulkarni R | Abdominal Wall Blocks in Abdominal Surgery: An Update | International Journal of Regional Anaesthesia | January-June 2021; 2(1): 47-53. 


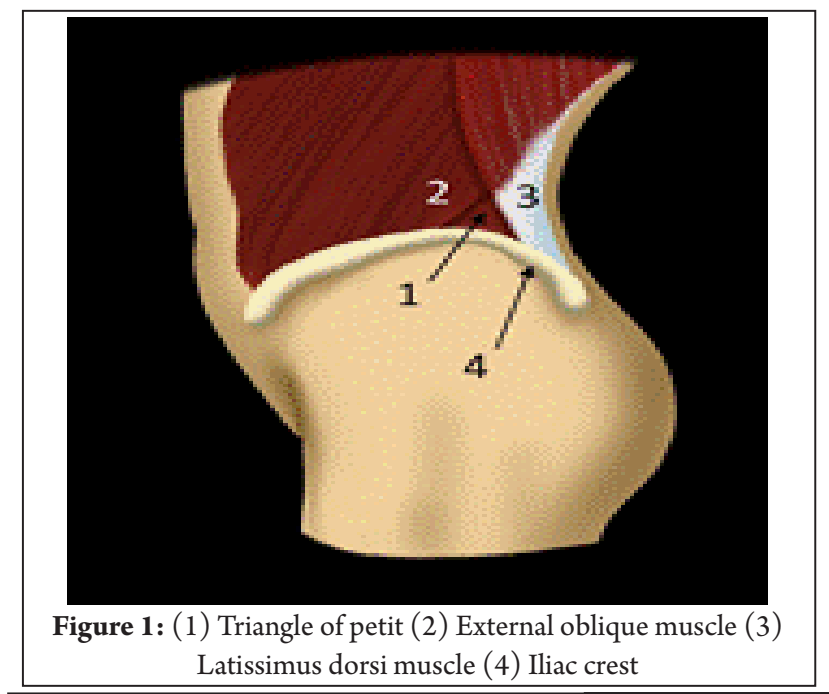

posteriorly to cover the anterior surfaces of the quadratus lumborum (QL) and the psoas major muscles (PMM). Quadratus lumborum (QL) muscle lies deep to the latissimus dorsi, anterolateral to the erector spinae muscle (ESM), and posterolateral to the TAM. It originates from the iliac crest and is inserted into the inferior border of the $12^{\text {th }} \mathrm{rib}$ and the transverse processes of the first to fourth lumbar vertebrae [8]. The muscles of the posterior abdominal wall include the QL and PMM, and are both considered hypaxial because they lie anterior to the transverse process [9].

The anterior rami of intercostal nerves T 7-12 (T12 also being called the subcostal nerve), which innervate the anterior abdominal wall along with the anterior rami of the first lumbar spinal nerve (L1) travel in the plane between the IOM and the TAM [7] (Fig. 3a). Posteriorly, the lumbar plexus lies in the

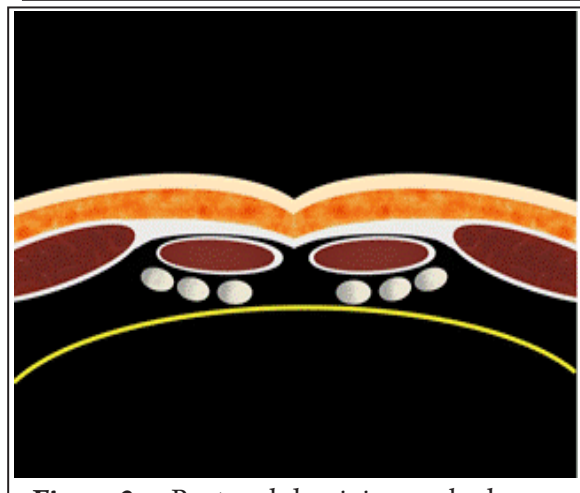

Figure 2 a: Rectus abdominis muscle above costal margin

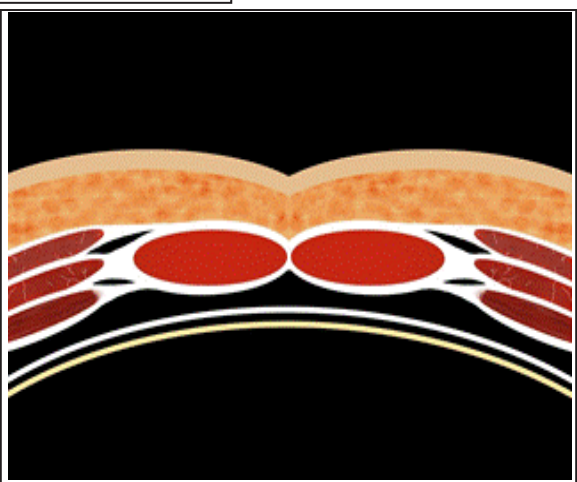

Figure 2 b: Rectus abdominis muscle \& sheath between costal margin \& arcuate line

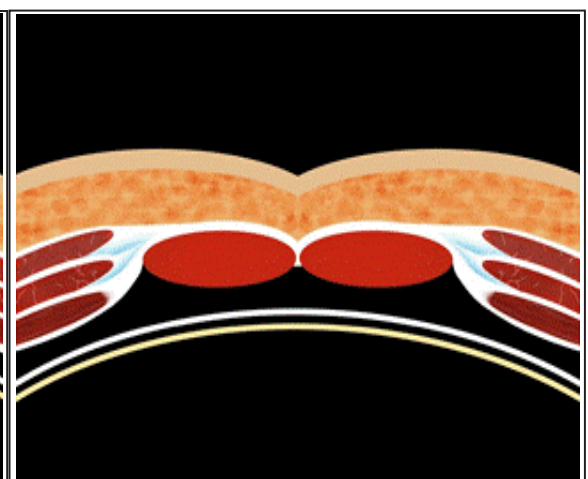

Figure 2 c: Rectus abdominis muscle \& sheath below arcuate line

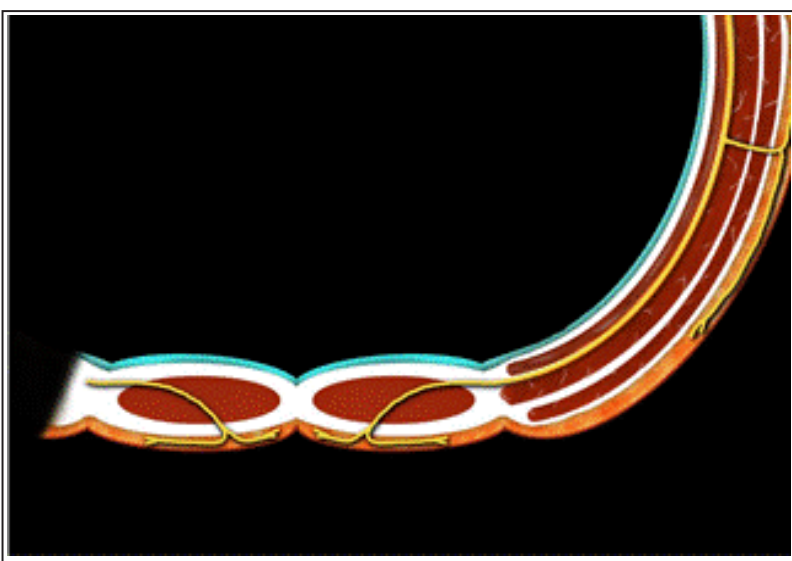

Figure 3 a: The Transversus abdominis plane (TAP)

the lower abdomen, the anterior sheath is formed by the aponeurosis of the external, internal, and transversus abdominis muscles. The posterior rectus sheath is absent below the arcuate line (a point about $1 / 3^{\text {rd }}$ the distance between the umbilicus and pubic crest). In this region, the posterior border of the rectus muscle is formed by the fascia transversalis (Figs. 2a, 2b, 2c) [7]. Bands of connective tissue, tendinous intersections, traverse RAM, which separates RAM into distinct muscle bellies anteriorly. The posterior rectus sheath is a continuous structure along the length of the muscle without intersections which facilitates caudo-cranial spread of the injectate.

Posteriorly, the IOM thins out to fuse with the lumbar fascia, while the transversalis fascia that lies inferior to the TAM continues

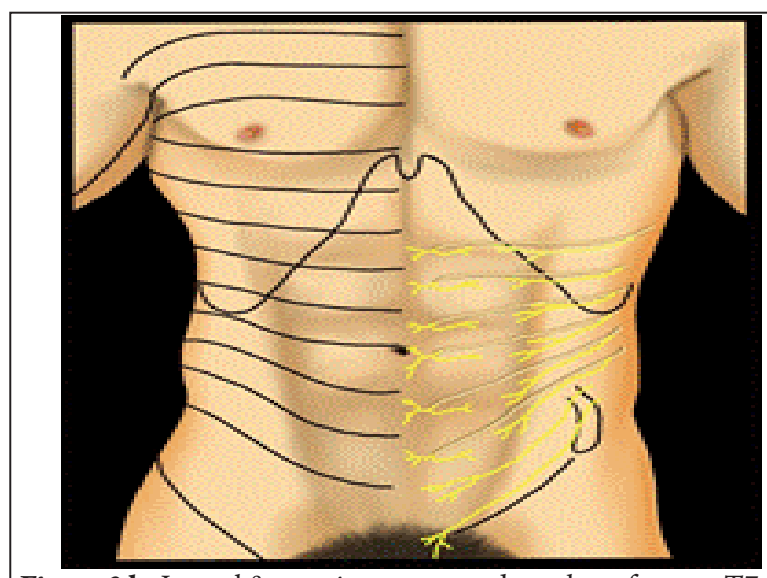

Figure 3 b: Lateral \& anterior cutaneous branches of nerves T7-12 \& L1

posterior thirds of the PMM, and the lower costal nerves travel in the plane between the QL and transversalis fascia and continue in the plane between the TAM and the IOM. After traveling in the anterior abdomen between the IOM and the TAM, the nerves pierce the posterior rectus sheath and lie underneath the muscle belly at the lateral half. They then traverse the muscle belly almost vertically to form the anterior cutaneous branches (Figs. 3a \& b). The inferior epigastric artery is also present between the rectus abdominis muscle and the posterior rectus sheath. This is of relevance as inadvertent puncture of this vessel could result in the formation of a hematoma. 


\section{Rectus Sheath Block}

In 1996, Ferguson described the use of the rectus sheath block to provide intra and postoperative analgesia in children undergoing umbilical hernia surgery [10]. With the advent of ultrasound, the rectus sheath block remains a simple and useful technique to provide reliable analgesia for midline incisions over the abdomen. Though popularly used to provide analgesia around the umbilicus (T9-T11), the rectus sheath block has also been used to provide analgesia for other midline incisions above the umbilicus such as for laparotomies [11]. Catheters can also be placed bilaterally to provide extended analgesia.

\section{Sonoanatomy and description of the ultrasound technique}

The rectus sheath block may be performed using a linear highfrequency probe. The probe is placed in the midline to identify the linea alba and is then moved laterally (on either side) to visualize the rectus muscle. The posterior rectus sheath is identified as a hyperechoic fascial line just under the belly of the rectus muscle (Fig. 4). Often, two parallel hyperechoic lines are seen below the muscle. The line closest to the muscle is the posterior rectus sheath, while the one below it is the fascia transversalis. Care should be taken to inject the local anaesthetic between the muscle and the posterior rectus sheath. $10-15 \mathrm{ml}$ of local anaesthetic is injected on either side. The inferior epigastric artery may be seen in this plane. The block must be done bilaterally to ensure midline analgesia.

\section{Transversus Abdominis Plane (TAP) Block}

The ultrasound guided transversus abdominis plane (TAP) block is now perhaps the most commonly performed abdominal wall block. The aim of the TAP block is to anaesthetize the seventh to the twelfth thoracic spinal nerves, as well as the first lumbar nerve. Though there are minor variants of the earliest described techniques, the overall concept is to place local anaesthetic between the internal oblique and the transversus abdominis muscle. Due to the extent of this plane, it is improbable that all the nerves present will come in contact with the local anaesthetic. Hence, the extent of spread is limited and may be inconsistent.

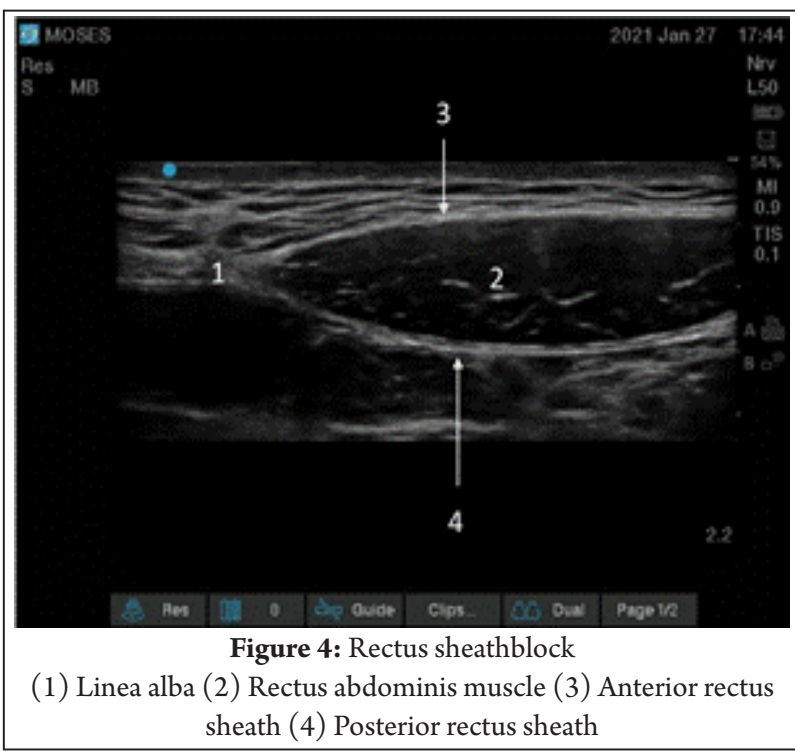

There are two different injection locations for this block to overcome this limitation. For analgesia above (and including) the umbilicus, (T10), the preferred technique is the 'Subcostal TAP Block'. For analgesia below the umbilicus, the "Classic TAP Block" is preferred. This rationale is derived from cadaver studies based on the spread of local anaesthetics in cadavers [12]. There is an overlap in nomenclature as well as the exact site of injection for the TAP block. Injections in the line of the anterior and midaxillary line can be best described as "Lateral TAP blocks". Injections that are more posterior to the midaxillary line, close to the border with the QL muscle are known as "posterior TAP blocks". Posterior injections have been shown to provide better analgesia than lateral injections [13].

The TAP block may be used for providing analgesia for a wide variety of commonly performed surgeries on the anterior abdominal wall. Some examples (but not limited to) arelaparotomies, open/lap appendectomy, caesarian section, open/lap cholecystectomy, renal transplants, and hernia repair. It is important to note that the TAP block only provides somatic analgesia and does not contribute to visceral analgesia. Therefore, the TAP block is best performed as part of a multimodal analgesic regimen.

\section{Sonoanatomy and description of the ultrasound technique for the TAP Block}

The ultrasound guided posterior TAP was originally described to provide analgesia below the umbilicus $[5,6]$. The block may be performed in a supine patient using a high frequency linear ultrasound probe. A systematic scan is initiated from the umbilicus and the probe is moved laterally. The aponeurosis of the EOM, IOM, and TAM are seen at the lateral edge of the rectus muscle. The probe is moved further lateral, following the contours of the abdomen till the lateral end of the TAM is visualized (Fig. 5). The appropriate needle entry trajectory tends to be almost $90^{\circ}$ to the anterior abdominal wall. This enables the proceduralist to achieve excellent images of the needle as well as a smooth entry into the plane. After the needle is inserted past the skin, it is guided through

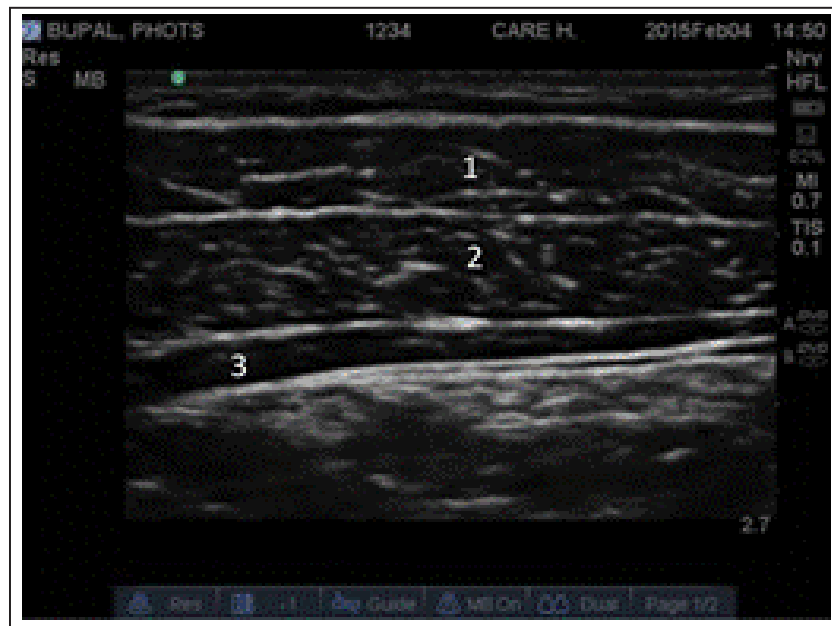

Figure 5: Lateral Conventional TAP block

(1) External obliquemuscle (2) Internal oblique muscle (3) Transversus abdominis muscle 
the external and internal oblique. The needle enters the transversus abdominis plane with a subtle 'pop'. A small amount of LA or saline is injected to confirm the appropriate placement of the needle between the internal oblique and transversus abdominis muscle. The block should be performed bilaterally for midline incisions. $20 \mathrm{ml}$ of LA in the permitted concentration on each side may be deposited in adults.

\section{Subcostal Variant of TAP Block}

\section{Sonoanatomy and description of the ultrasound technique}

The subcostal TAP was described to overcome the limitations of the posterior TAP block in the area above the umbilicus [14]. The scan is started close to the xiphisternum and the probe is angled towards the costal margin. The rectus abdominis is identified and the TAM is usually seen under the lateral border of the rectus. As the probe moves further lateral, the aponeurosis of the external and internal oblique muscle is visualized and subsequently, the three muscles of the abdominal wall are seen stacked upon each other. It is favorable to initiate hydro dissection posterior to the rectus muscle in the plane between the posterior rectus sheath and TAM (Fig. 6). Using a long block needle (100-150 mm), the needle can be directed to traverse the dissected plane. This involves moving the needle further lateral along the costal margin till the needle tip lies between the internal oblique and the transversus abdominis muscle. Another alternative is to move the probe further lateral and perform a second needle puncture to deposit additional local anaesthetic in which case it is called the 'dual'block [8].

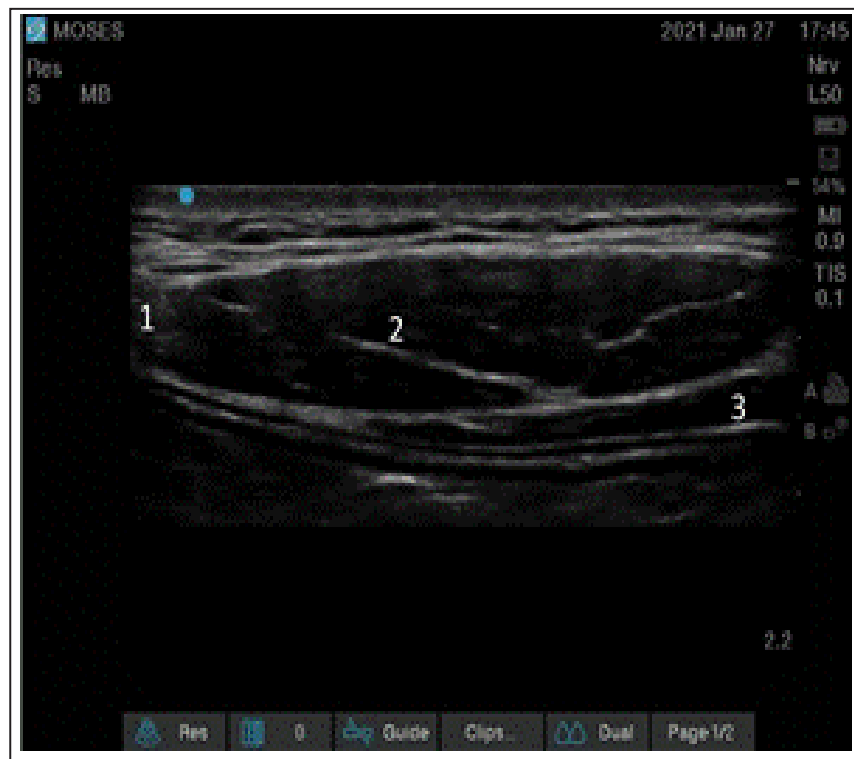

Figure 6: Subcostal TAP block

(1) Linea alba (2) Rectus abdominis muscle (3) Transversus abdominis muscle

\section{Ilioinguinal/Iliohypogastric Nerve Block}

Regional blockade of the ilioinguinal and iliohypogastric nerves in the abdomen can provide analgesia for surgeries performed in the inguinal region. Both these nerves are branches of the $\mathrm{L} 1$ nerve root and primarily supply the skin and abdominal muscles in the inguinal region. Occasionally, these nerves may originate from
T12 [15]. The nerves pierce the transversus abdominis muscle just superomedial to the anterior superior iliac spine (ASIS) and lie in the transversus abdominis plane. The nerves are usually accompanied by a branch of the deep circumflex iliac artery. The ilioinguinal nerve also innervates the inguinal hernia sac, the medial aspect of the thigh, and the anterior scrotum. In females, it supplies the labia.

\section{Sonoanatomy and description of the ultrasound technique}

A high frequency linear ultrasound probe is commonly used as this is a superficial block. The probe is placed on the ASIS of the side that requires the regional block in a transverse plane. Practitioners familiar with the TAP block should note that the nerves lie in the same plane. However, due to its peripheral attachments, the external oblique muscle may appear very thin in this area. The nerves are seen as hypoechoic structures, usually accompanied by a branch of the deep circumflex iliac artery, that may be visualized by using the color mode on the ultrasound machine (Fig. 7) 5-10 $\mathrm{ml}$ of LA is sufficient to provide anaesthesia/analgesia.

\section{Transversalis Fascia Plane Block}

The transversalis fascia block was described as a technique to block the lateral cutaneous branches of the L1 nerve, primarily to provide analgesia to the iliac crest in bone graft harvest surgery [16]. Local anaesthetic is deposited under the transversus abdominis muscle at its lateral edge and superficial to the fascia transversalis, close to the iliac crest (Fig. 8). This is similar to the QL 1 block. There is currently very little evidence on the utility of this block beyond bone graft harvest surgeries.

\section{Quadratus Lumborum Block}

Quadratus lumborum block (QLB) was first described by Rafael Blanco in 2007. This block is unique from other abdominal wall blocks in that both somatic and visceral analgesia are provided. The latter is likely to be possible owing to the communication of layers of thoracolumbar fascia (TLF), which, according to more common terminology, has three layers, anterior, middle and posterior, with the endothoracic fascia posterior to the diaphragm via medial and lateral arcuate ligaments and aortic hiatus [17]. Recent nomenclature presents four distinct techniques, namely QL 1 (lateral), QL 2 (posterior)targeting the lumbar interfascial triangle, LIFT, QL 3 (transmuscular or anterior) described by Borglum et al in 2013, and QL 4 (intramuscular) introduced by Murouchi, each of which has a different approach and injection endpoint (Fig. 9) [17, 18, 19]. Both single shot and continuous QLB are practiced, however, at this point of time, there is no scientific evidence that establishes the superiority of one approach over the other, nor is the fate of a catheter placed in the TLF conclusively determined $[20,21]$. Given the proximity of the lumbar plexus and the continuation of the anterior TLF caudally with the fascia iliaca, blockade of lumbar plexus is likely, and clinical reports suggest QL block for analgesia following hip surgery. Pre-emptive QLB in conjunction with general anaesthesia can reduce intraoperative analgesic requirement. Unilateral QLB can be used to provide postoperative analgesia in unilateral abdominal surgeries, such as nephrectomy, whereas bilateral QLB can be employed for a wide variety of abdominal surgeries for postoperative analgesia [8]. 


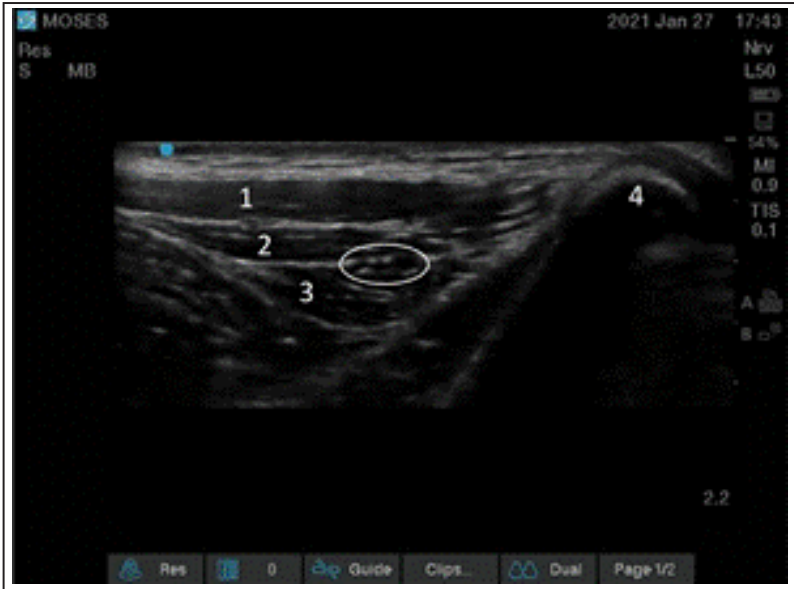

Figure 7: Ilioinguinal \& Iliohypogastric nerve blocks

(1) External oblique muscle (2) Internal Oblique Muscle (3)

Transversus abdominis muscle (4) Ant. Sup. Iliac spine Oval outline highlights 3 hypoechoic rings, far left is ilioinguinal nerve, centre is ascending branch of circumflex iliac artery, far right is iliohypogastric nerve

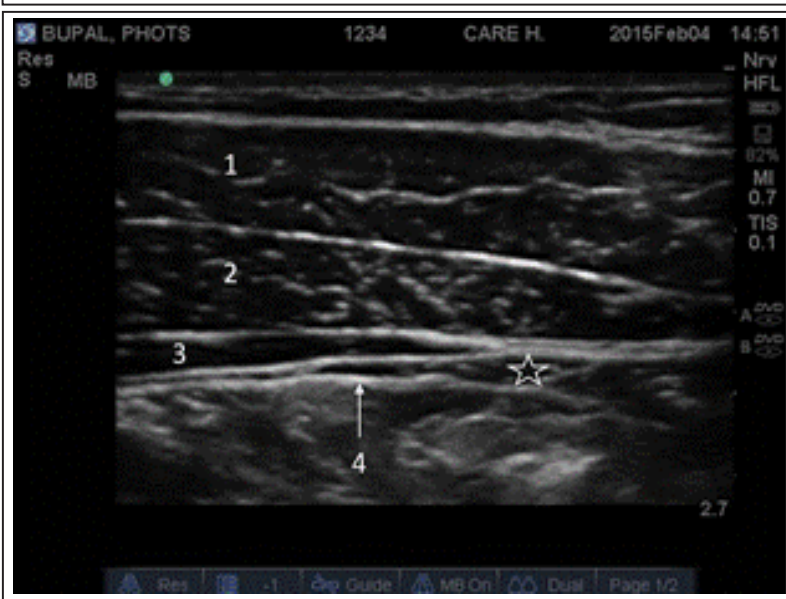

Figure 8: Transversalis fascia plane block

(1) External oblique muscle (2) Internal Oblique muscle

(3) Transversus abdominal muscle

(4) Transversalis fascia

Star: location for deposition of LA

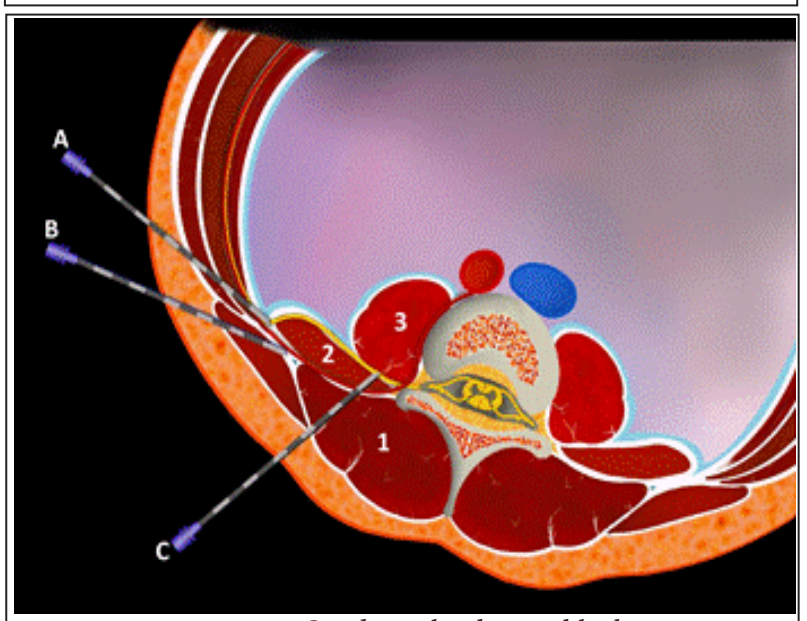

Figure 9: Quadratus lumborum block

(1) Erector spinae muscle (2) Quadratus lumborum muscle (3) Psoas major muscle (3 A) QL 1 or lateral approach (3 B) QL 11 or posterior approach (3 C) QL 111 or anterior apporach

\section{Complication of Abdominal wall Blocks}

The commonest concern with abdominal wall blocks is the puncture of the peritoneal cavity, including abdominal organs and vasculature. Trauma to the liver has occurred with TAP blocks [22, 23]. Intraperitoneal bowel hematoma has been reported with ilioinguinal-iliohypogastric nerve blocks [24]. Retroperitoneal hematoma has been described after rectus sheath block [25]. There are no reports of neurological injuries after abdominal wall blocks. This is likely to be due to the targeting of planes, rather than neural structures. It must be noted that the advent of ultrasound along with appropriate techniques should minimize, if not eliminate the above complications which are likely to be due to technical issues rather than the block itself. Local anaesthetic plasma levels have been shown to reach high levels after ultrasound guided TAP blocks with ropivacaine $[26,27]$. This is likely due to the large volumes of LA used as well as the spread across the muscle surface. The inadvertent spread of the local anaesthetic to unintended sites has also been reported. Femoral nerve block has been reported after ilioinguinal-iliohypogastric blocks $[28,29]$ as well as the TAP block [30]. The transmuscular QLB (anterior) is best avoided in coagulopathic patients.

\section{Evidence Basis for Abdominal Wall Blocks}

Though the rectus sheath block has been used for surgical anaesthesia in umbilical hernia repair [31], it is unclear if it is superior to local infiltration techniques [32]. Using continuous catheter techniques, rectus sheath blocks have been shown to provide effective analgesia in midline laparotomies [33, 34]. A meta-analysis of the analgesic effects of TAP block in abdominal surgeries showed that there are marginal post-operative analgesic benefits. There are variations in the technique as well as the volume of local anaesthetic used $(12-30 \mathrm{ml} /$ side and these may influence the analgesic effects [35]. Preoperative blocks may offer better outcomes than those administered postoperatively and there is an overall reduction in morphine consumption when used for laparoscopic surgery [36]. TAP blocks have been studied extensively for caesarian sections and are well known to reduce pain when used in patients who have not received intrathecal opioids [37]. Ilioinguinal-iliohypogastric nerve blocks have an established history for hernia and orchidopexy. Though used for somatic analgesia, the analgesia provided after pediatric inguinal hernia surgery may be comparable to that achieved by caudal analgesia $[38,39]$, but may not be superior to local infiltration techniques [40]. Two RCTs on the QL2 (posterior) block demonstrated that there was a significant reduction in opioid consumption and pain scores for up to 48 hours when added to a multimodal analgesic regimen $[41,42]$. Reduced post-operative pain scores were seen in laparoscopic gynaecological procedures and lower abdominal surgeries [43, 44]. There have been several reports of the use of the quadratus lumborum block in surgeries that extend as high as T6, thereby triggering the postulation of many theories on the spread and mechanism of action [17]. When compared with epidural analgesia for laparoscopic nephrectomy, the QLB showed comparable analgesia [46]. 


\section{Conclusions}

Ultrasound guided abdominal wall blocks are an option for postoperative analgesia after abdominal surgeries and offer a viable alternative to the invasive and often technically challenging continuous thoracic epidural analgesia and thoracic paravertebral blocks. With increasing experience and an understanding of clinical and sonoanatomy, the regional anaesthesiologist is enabled to select the right abdominal wall block for the surgical procedure, reduce block performance time and complications.
The QLB is unique in that, besides somatic analgesia, visceral analgesia is afforded. However, optimal injection endpoint, volume of local anaesthetic, predictable spread of drug and fate of the QL catheter will require further work for better definition of this block. Though the primacy of thoracic epidural analgesia as gold standard for pain relief after abdominal surgery is unlikely to be challenged, ultrasound guided abdominal wall blocks will gain wider acceptance in clinical practice.

\section{References}

1. Susan M Nimmo, Lorraine S Harrington. What is the role of epidural analgesia in abdominal surgery? Continuing Education in Anaesthesia, Critical care and Pain 2014, Vol.14.

2. Schliech D. Schmerzlose Operationen. In Springer Verlag; 1899.p.240-58 (4th edition)

3. Thomas H Seldon. Regional anesthesia for surgery of the thorax and abdominal wall. Section on Anesthesia 1941, Mayo Clinic, Rochester, Minnesota; Vol.2.

4. Rafi AN. Abdominal field block; a new approach via the lumbar triangle. Anaesthesia 2001; 56(10): 1024-6

5. Mc Donnell JG, O'Donnell BD, Farrell T et al. Transversus abdominis plane block; a cadaver and radiological evaluation. Reg Anesth Pain med.2001; 32;399-404.

6. Hebbard P, Fujiwara Y, Shibata Y, Royse C. ultrasound-guided transversus abdominis plane (TAP) block. Anaesth Intensive care 2007 Aug; 35(4): 616-7.

7. Ki Jinn Chin, John G McDonnell, Brendan Carvalho et al. essentials of our current understanding: abdominal wall blocks. Reg Anesth Pain Med 2017; 42 : 133-183.

8. DN Onwochei, J Borglum, A Pawa. Abdominal wall blocks for intra-abdominal surgery. BJAEducation 2018; Vol.18, No.10.

9. FH Willard, A Vleeming, MD Schuenke et al. the thoracolumbar fascia : anatomy, function and clinical considerations. Journal of Anatomy 2012; 221(6): 507-536.

10. Ferguson S, Thomas V, Lewis I. the rectus sheath block in pediatric anaesthesia : new indicationsfor an old technique?Pediatric Anesthesia 1996 Dec; 6(6): 463-6.

11. Melesse DY, Chekol WB, Tawuye HY, Denu ZA et al. Assessment of the analgesic effectiveness of rectus sheath block in patients who had an emergency midline laparotomy : prospective observational cohort study. International Journal of Surgery open 2020; 24 $: 27-31$.

12. Lee THW, Barrington MJ, Tran TNM, Wong D, Hebbard P. Comparison of extent of sensory block following posterior and subcostal approaches to ultrasound-guided transversus abdominis plane block. Anaesthesia and Intensive care 2010 May; 38(3): $452-460$.

13. Yoshiyoma S, Ueshima H, Sakai R, Otake H. A posterior TAP block provides more effective analgesia than a lateral TAP block in patients undergoing laparoscopic gynaecologic surgery: a retrospective study. Anesthesiology Research and Practice 2016; 2016:1-5.

14. Hebbard P. Subcostal transversus abdominis plane block under ultrasound guidance. Anesthesia and Analgesia 2008; 106(2).

15. Klaassen Z, Marshall E, Tubbs RS, Louis RGJ et al. Anatomy of the ilioinguinal and iliohypogastric nerves with observations of their spinal nerve contributions. Clin Anat. 2011 May; 24(4): 454-61.

16. Hebbard P. Transversalis fascia plane block, a novel ultrasound guided abdominal wall nerve block. CanJ Anesth. 2009Aug; 56(8):618-20.

17. Hesham Elsharkawy, Kariem El-Boghdadly, Michael Barrington. Quadratus lumborum block: anatomical concepts, mechanisms and techniques. Anesthesiology Feb 2019; Vol.130, No.2.

18. Sauter AR, Ullensvang K, Niemi G, Lorentzen HT, Bensten TF, Borglum J. The shamrock lumbar plexus block : a dose finding study. Eur J Anaesthesiol 2015; 32 : 764 70 .
19. Murouchi T. Quadratus lumborum block intramuscular approach for pediatric surgery. Acta Anesthesiologica Taiwanica 2016.

20.TVS Gopal. Ultrasound guided transmuscular quadratus lumborum plane catheters : in the plane or out of it ?Editorial-IndianJAnaesth 2019; 63:609-10.

21. Diwan S, Kulkarni M, Kulkarni N, Nair A. A radiological inquest to determine the destiny of ultrasound guided transmuscular quadratus lumborum plane catheters. Indian J Anaesth 2019; 63: 667-70.

22. Lancaster $P$, Chadwick M. Liver trauma secondary to ultrasound-guided transversus abdominis plane block. BrJAnaesth. 2010 Apr;104(4):509-10.

23. Farooq $M$, Carey M. A case of liver trauma with a blunt regional anesthesia needle while performing transversus abdominis plane block. Reg Anesth Pain Med. 2008 Jun;33(3):274-5.

24. Frigon C, Mai R, Valois-Gomez T, Desparmet J. Bowel hematoma following an iliohypogastric-ilioinguinal nerve block. Paediatr Anaesth. 2006 Sep;16(9):993-6.

25. Yuen PM, Ng PS. Retroperitoneal hematoma after a rectus sheath block. J Am Assoc Gynecol Laparosc. 2004Nov;11(4):448.

26. Griffiths JD, Barron FA, Grant S, Bjorksten AR, Hebbard P, Royse CF. Plasma ropivacaine concentrations after ultrasound-guided transversus abdominis plane block. BrJAnaesth.2010Dec;105(6):853-6.

27. Hessian EC, Evans BE, Woods JA, Taylor DJ, Kinkel E, Bjorksten AR. Plasma ropivacaine concentrations during bilateral transversus abdominis plane infusions. British Journal of Anaesthesia. 2013 Sep 1;111(3):488-95.

28. Shivashanmugam T, Kundra P, Sudhakar S. Iliac compartment block following ilioinguinal iliohypogastricnerve block. Paediatr Anaesth. 2006 Oct;16(10):1084-6.

29. Ghani KR, McMillan R, Paterson-Brown S. Transient femoral nerve palsy following ilio-inguinal nerve blockade for day case inguinal hernia repair. J R Coll Surg Edinb. 2002 Aug;47(4):626-9.

30. Manatakis DK, Stamos N, Agalianos C, Karvelis MA, Gkiaourakis M, Davides D. Transient femoral nerve palsy complicating "blind" transversus abdominis plane block. Case reportsin anesthesiology. 2013;2013:874215.

31. Quek KHY, Phua DSK. Bilateral rectus sheath blocks as the single anaesthetic technique for an open infraumbilical hernia repair. Singapore Med J. 2014 Mar;55(3):e39-41.

32. Rajwani KM, Butler S, Mahomed A. In children undergoing umbilical hernia repair is rectus sheath block effective at reducing post-operative pain? Best evidence topic (bet). International Journal of Surgery. 2014;12(12):1452-5.

33. Tudor ECG, Yang W, Brown R, Mackey PM. Rectus sheath catheters provide equivalent analgesia to epidurals following laparotomy for colorectal surgery. Ann $R$ Coll Surg Engl. 2015 Oct; $97(7): 530-3$.

34. Godden AR, Marshall MJ, Grice AS, Daniels IR. Ultrasonography guided rectus sheath catheters versus epidural analgesia for open colorectal cancer surgery in a single centre. Ann R Coll SurgEngl. 2013 Nov;95(8):591-4.

35. Baeriswyl M, Kirkham KR, Kern C, Albrecht E. The Analgesic Efficacy of Ultrasound-Guided Transversus Abdominis Plane Block in Adult Patients: A MetaAnalysis. Anesthesia \& Analgesia. 2015 Dec;121(6):1640-54. 
36. De Oliveira GS, Castro-Alves LJ, Nader A, Kendall MC, McCarthy RJ. Transversus Abdominis Plane Block to Ameliorate Postoperative Pain Outcomes After Laparoscopic Surgery: A Meta-Analysis of Randomized Controlled Trials. Anesthesia \& Analgesia. 2014Feb;118(2):454-63.

37. Mishriky BM, George RB, Habib AS. Transversus abdominis plane block for analgesia after Cesarean delivery: a systematic review and meta-analysis. Can J Anesth/JCanAnesth.2012 Aug;59(8):766-78.

38. Hannallah RS, Broadman LM, Belman AB, Abramowitz MD, Epstein BS. Comparison of caudal and ilioinguinal/iliohypogastric nerve blocks for control of postorchiopexy pain in pediatric ambulatory surgery. Anesthesiology. 1987 Jun;66(6):832-4.

39. Markham SJ, Tomlinson J, Hain WR. Ilioinguinal nerve block in children. A comparison with caudal block for intra and postoperative analgesia. Anaesthesia. 1986 Nov;41(11):1098-103.

40. Reid MF, Harris R, Phillips PD, Barker I, Pereira NH, Bennett NR. Day-case herniotomy in children. A comparison of ilio-inguinal nerve block and wound infiltration for postoperative analgesia. Anaesthesia. 1987Jun;42(6):658-61.
41. Blanco R, Ansari T, Girgis E. Quadratus lumborum block for postoperative pain after caesarean section: A randomised controlled trial. Eur J Anaesthesiol. 2015 Nov;32(11):812-8.

42. Mieszkowski MM, Mayzner-Zawadzka E, Tuyakov B, Mieszkowska M, Żukowski M, Waśniewski T, et al. Evaluation of the effectiveness of the Quadratus Lumborum Block type I using ropivacaine in postoperative analgesia after a cesarean section - a controlled clinicalstudy. Ginekol Pol.2018;89(2):89-96.

43. Ishio J, Komasawa N, Kido H, Minami T. Evaluation of ultrasound-guided posterior quadratus lumborum block for postoperative analgesia after laparoscopic gynecologic surgery.JClin Anesth.2017Sep;41:1-4.

44. Öksüz G, Bilal B, Gürkan Y, Urfalioğlu A, Arslan M, Gişi G, et al. Quadratus Lumborum Block Versus Transversus Abdominis Plane Block in Children Undergoing Low Abdominal Surgery: $<e m>A$ Randomized Controlled Trial</em $>$. Reg Anesth Pain Med.2017Sep 1;42(5):674.

45. Aditianingsih D, Pryambodho, Anasy N, Tantri AR, Mochtar CA. A randomized controlled trial on analgesic effect of repeated Quadratus Lumborum block versus continuous epidural analgesia following laparoscopic nephrectomy. BMC Anesthesiology. 2019Dec5;19(1):221.

\section{Conflict of Interest: Nil Source of Support: None}

